\title{
THE EXTRAGALACTIC REFERENCE FRAME
}

\author{
Dennis D. McCarthy (USNO) \\ Jane Russell (NRL) \\ Brent Archinal (USNO) \\ Merri sue Carter (USNO) \\ Dave Florkowski (USNO) \\ Ellis Holdenried (USNO) \\ Ken Johnston (NRL) \\ Zhen-Guo Yao (USNO)
}

ABSTRACT. A cooperative program has been established to produce a reference frame of at least 400 suitable extragalactic sources. This is an extension of collaborative efforts (Johnston et al., Russell et al., Ma et al., and Reynolds et al.) reported at this meeting. In Phase one, a catalog of radio source positions will be constructed using original VLBI observations already obtained by NASA, NGS, NRL, JPL and USNO. Observations will be re-reduced in a consistent system to avoid some of the more serious problems associated with the formation of compilation catalogs. In Phase Two, the USNO will maintain the system, including the monitoring of source structure and/or variation. During both phases cooperation among the various agencies will continue. A list of sources will be made available to optical observers so that the frame will have benchmarks in both the optical and radio regimes.

\section{Introduction}

The IAU Working Group on the Radio/Optical Reference Frame was established in recognition of the importance of an extragalactic radio reference frame to the improvement of the optical one. In Argue et al. (1984), the working group published a list of 233 sources with positions compiled from several published radio catalogs as a starting point. The importance of the completion and maintenance of the Extragalactic Reference System catalog was emphasized at a recent workshop on the Celestial Reference system sponsored by the National Earth orientation Service and held at the $U$. S. Naval Observatory (USNO). While all agreed on the importance of an on-going reference frame program and all had made observations which were useful for it, none of the agencies represented had long term plans for maintaining the catalog for astronomy. 
The current Navy program (Johnston, et al., 1988), is the only active program whose goal is to produce an extragalactic radio frame suitable for ties to the other astronomical reference frames, although others have announced plans toward the same goal. The Navy program incorporates some data which had been obtained previously for other purposes (e.g. Crustal Dynamics Program (CDP), IRIS) and new data obtained solely for the reference frame project, all with the cooperation of individuals from several agencies (NRL, USNO, NASA/CDP, NASA/JPL, NOAA/NOS/NGS) and countries (USA, Australia, Germany, Japan, South Africa). Optical positions of the sources having optical counterparts are also being measured as part of the current program. In accordance with the goal of providing a radio/optical tie-in, the RA zero point for the extragalactic radio reference frame is fixed using positions of optical counterparts of 28 quasars in the system of the FK5.

The aim of the Navy program is to establish a reference system of at least 400 extragalactic sources (about one per 100 square degrees), which are compact, have a flat spectrum in the radio, display optical emission, and are evenly distributed about the sky. It began in 1987 with a 5-yr plan. As of September, 1989,347 sources have been observed in VLBI geodetic or astrometric experiments. The maintenance of the large data bases for this work have been done as a cooperative effort with the NASA Crustal Dynamics Project, scheduled to end in December 1991.

The radio positions published so far are in $\mathrm{Ma}$, et al. (1990, 182 sources) and Russell, et al. (1991a, 55 more; $1991 \mathrm{~b}, 39$ more for a total of 276 published sources). At a recent workshop on the Celestial Reference system sponsored by the National Earth orientation Service and held at the Naval observatory participants concluded that a standard VLBI reference system should be created, maintained and related to other systems. While other catalogs of radio source positions exist, none are comprehensive and most were created for other purposes, solving for various other parameters in their derivations. The proposed catalog would fill this need for the entire astronomical community.

\section{Objectives}

The object of this program is to assume responsibility for the production and maintenance of a standard celestial reference frame defined by VLBI observations of extragalactic radio sources. This project will be a joint USNO/NRL project with extensive cooperation among other agencies (NASA/GSFC, NGS, NASA/JPL, CSIRO, and Hamberger sternwarte). At the beginning of the project much of the work will be performed at NRL but transition to operation at USNO will begin immediately. 
Complete transition to an operational USNO program will occur at the beginning of 1993. The project can be considered to be divided into two separate phases.

Phase one is the initialization of the radio program at USNO. This will consist of establishing a data base with all available dual frequency Mark III VLBI data at USNO. This will be the crustal Dynamics Data Base with small modifications for precise astrometric analysis or something very similar to the CDP data base. The software necessary to reduce this data currently used in the Navy program will be reviewed with the aim of establishing a uniform set of software for the program. There are two objectives in Phase One. First, an initial catalog of source candidates and source positions from the present data base will be chosen. This work will supercede the Argue et al. list and give a preliminary reference frame. Second, a first catalog of radio source positions of all 400 sources and alternates will be produced.

This First Radio Reference Frame catalog will be reduced in a consistent system by NRL/USNO personnel using all the collected data in the data base to avoid some of the problems associated with previous compilation catalogs. In Phase one of the program, the universal radio data base will be established at USNO and the software algorithms necessary to produce excellent astrometric results from this data base will be verified. Observations will be ongoing. Phase one is expected to end in 1992 .

In Phase Two, following the initial catalog, the USNO will make VLBI observations to densify and maintain the system. The monitoring of source structure and/or variation will be carried out by NRI/USNO. Most of these observations would be made in conjunction with routine observations made for the determination of Earth orientation. Use of the VLBA by NRL/USNO would be anticipated to investigate possible source structure problems. Observations required to improve celestial reference frame ties would continue to be made by USNO/NRL. These might include, for example, observations of radio stars in radio and optical wavelengths, optical observations of quasars, radio observations of solar system objects, millisecond pulsar observations, etc. During both phases, cooperation among the various agencies would continue.

\section{3. specifications}

The VLBI reference frame will consist of the positions of approximately 400 sources distributed evenly in the sky (Figure 1). The accuracy of these positions exceed the level of \pm 1 millisecond of arc. The frame will be updated annually with announcements of problems which may be caused by varying source structure or brightness. Complete revisions of the 
system will occur approximately every five years. The zero point of the right ascension coordinate will be specified by the IAU working group on origins. The pole is to be defined by the IERS pole or an alternate proposed by the IAU Working Group on Reference Systems. IERS standards will be used in the solutions unless specified otherwise. If other values for critical constants or procedures are used other than the IERS values, they will be described in full detail.

\section{Conclusions}

The proposed catalog could serve as the radio source catalog for use as the extragalactic reference frame. Phase one, to be completed in 1992, will produce a catalog of 400 sources all reduced in the same system. Phase Two will continue this work with periodic updates.

\section{References}

Argue, A.N., de Vegt, C., Elsmore, B., Fanselow, J., Harrington, R., Hemenway, P., Johnston, K.J., Kuehr, H., Kumkova, I., Neill, A.E., Walter, H., Witzel, A.: 1984, Astron. Astrophys. 130, 191.

Johnston, R.J., Russell, J., de Vegt, C., Hughes, J., Jauncey, D., White, G., and Nicholson, G.: 1988, in The Impact of VLBI on Astrophysics and Geophysics, IAU Symp. 129, M. Reid and J. Moran, eds., (Reidel, Dordrecht), 317.

Ma, C., Shaffer, D.B. de Vegt, Chr., Johnston, K.J., Russell, J.L.: 1990, Astron. J. 99, 1284 .

Russell, J.L., Johnston, K.J., Ma, C., Shaffer, D., devegt, C.: 1991a, Astron. $J$. in press.

Russell, J.L., Johnston, K.J., Jauncey, D., White, G., Reynolds, J., Harvey, B., Nothnagel, A., Nicholson, G., Kingham, K., Ma, C., de Vegt, C., Hindsley, R., Zacharias, N.: $1991 \mathrm{~b}$, in preparation. 


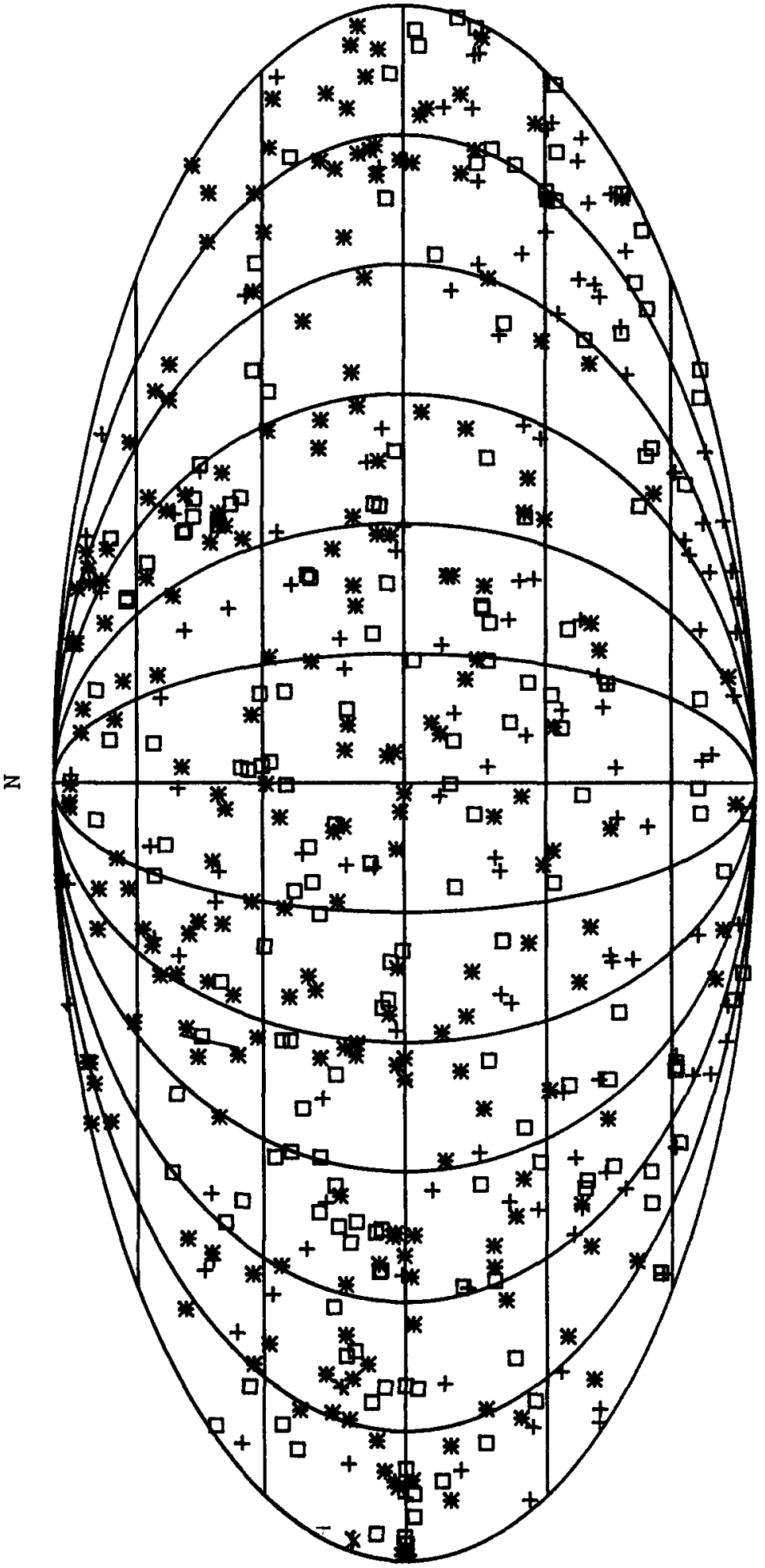

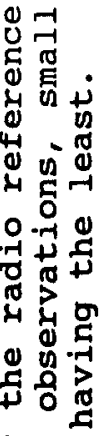
넝복 (1) $>$ ) -4 o न

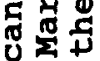

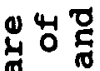
대 4

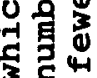
乌ั H อ o 9 40 0 出

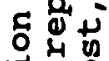
+口 ว

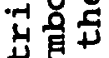

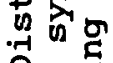
Q 1$)^{-n}$ . E -

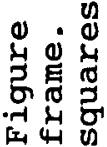

